\title{
Modification in CSF specific gravity in acutely decompensated cirrhosis and acute on chronic liver failure independent of encephalopathy, evidences for an early blood-CSF barrier dysfunction in cirrhosis
}

\author{
Nicolas Weiss * ${ }^{1,2,3}$, Matteo Rosselli ${ }^{*}$, Sarah Mouri ${ }^{1,5}$, Damien Galanaud ${ }^{6}$, Louis Puybasset ${ }^{7}$, \\ Banwari Agarwal ${ }^{4}$, Dominique Thabut ${ }^{1,5}$ and Rajiv Jalan ${ }^{4}$ \\ * the authors contributed equally to the work \\ 1 Brain-Liver Pitié-Salpêtrière Study Group (BLIPS), Hôpital de la Pitié Salpétrière, Assistance \\ Publique-Hôpitaux de Paris, Paris, France \\ 2 Unité de réanimation neurologique, Fédération de Neurologie 1, Pôle des maladies du système \\ nerveux, Hôpital de la Pitié Salpêtrière, Assistance Publique-Hôpitaux de Paris, Paris, France \\ ${ }^{3}$ Institut de Neurosciences Translationnelles de Paris, Institut-Hospitalo-Universitaire-A-Institut du \\ Cerveau et de la Moelle (IHU-A-ICM), Paris, France \\ ${ }^{4}$ Liver Failure Group, UCL Institute for Liver and Digestive Health, UCL Medical School, Royal Free \\ Hospital, London \\ ${ }^{5}$ Soins Intensifs d'Hépatologie, Service d'Hépato-Gastroentérologie, Hôpital de la Pitié Salpétrière, \\ Assistance Publique-Hôpitaux de Paris, Paris, France \\ ${ }^{6}$ Service de Neuroradiologie, Hôpital de la Pitié-Salpêtrière, Assistance Publique - Hôpitaux de Paris, \\ Paris, France \\ 7 Neuro-réanimation chirurgicale, département d'anesthésie-réanimation, Hôpital de la Pitié- \\ Salpêtrière, Assistance Publique - Hôpitaux de Paris, Paris, France and Université Pierre et Marie \\ Curie
}

\section{Correspondance :}

Nicolas WEISS, MD, PhD

Brain Liver Pitié-Salpêtrière (BLIPS) study group

Unité de Réanimation Neurologique

Neurologie 1, pole des maladies du système nerveux

Groupement Hospitalier Pitié-Salpêtrière-Charles Foix

Assistance Publique - Hôpitaux de Paris, Paris, France

Phone: $\quad 0142162770$

Fax: $\quad 0142161989$

nicolas.weiss@psl.aphp.fr 


\section{ABSTRACT}

Introduction: Although hepatic encephalopathy (HE) on the background of acute on chronic liver failure (ACLF) is associated with high mortality rates, it is unknown whether this is due to increased blood-brain barrier permeability. Specific gravity of cerebrospinal fluid measured by CT is able to estimate blood-cerebrospinal fluidbarrier permeability. This study aimed to assess cerebrospinal fluid specific gravity in acutely decompensated cirrhosis and to compare it in patients with or without ACLF and with or without hepatic encephalopathy.

Method: We identified all the patients admitted for acute decompensation of cirrhosis who underwent a brain CT-scan. Those patients could present acute decompensation with or without ACLF. The presence of hepatic encephalopathy was noted. They were compared to a group of stable cirrhotic patients and healthy controls. Quantitative brain CT analysis used the Brainview software that gives the weight, the volume and the specific gravity of each determined brain regions. Results are given as median and interquartile ranges and as relative variation compared to the control/baseline group.

Results: 36 patients presented an acute decompensation of cirrhosis. Among them, 25 presented with ACLF and 11 without ACLF; 20 presented with hepatic encephalopathy grade $\geq 2$. They were compared to 31 stable cirrhosis patients and 61 healthy controls. Cirrhotic patients had increased cerebrospinal fluid specific gravity (CSF-SG) compared to healthy controls $(+0.4 \%, p<0.0001)$. Cirrhotic patients with ACLF have decreased CSF-SG as compared to cirrhotic patients without ACLF ($0.2 \%, p=0.0030)$ that remained higher than in healthy controls. The presence of hepatic encephalopathy did not modify CSF-SG $(-0.09 \%, p=0.1757)$. Specific gravity 
did not differ between different brain regions according to the presence or absence of either ACLF or HE.

Conclusion: In patients with acute decompensation of cirrhosis, and those with ACLF, CSF specific gravity is modified compared to both stable cirrhotic patients and healthy controls. This pattern is observed even in the absence of hepatic encephalopathy suggesting that blood-CSF barrier impairment is manifest even in absence of overt hepatic encephalopathy.

Key words: hepatic encephalopathy; brain edema; blood-brain barriers; bloodcerebrospinal fluid-barrier; CT scan 


\section{INTRODUCTION}

Overt hepatic encephalopathy $(\mathrm{HE})$ requiring hospital admission is a common complication of cirrhosis that is associated with high resource utilization and a significant in-hospital mortality $[1,2]$. HE patients have a significantly lower survival compared with patients without HE at all severities of liver disease [3,4]. Acute on chronic liver failure (ACLF) is a clinically and prognostically distinct syndrome that occurs in cirrhotic patients who are hospitalized with acute deterioration due to either known or unknown precipitating events, hepatic and/or extrahepatic organ failure and high short term mortality rates. Pathophysiologically, the syndrome is characterized by altered host response to injury. One major recent finding is that HE in patients displaying a diagnosis of ACLF have higher mortality compared with HE patients without ACLF [3].

The mechanism underlying the pathophysiology of HE in ACLF and this susceptibility to increased mortality is not known. In spite of its high frequency, HE pathophysiology is poorly understood. Increased cerebral ammonia uptake, and its transformation into glutamine by the astrocytes are thought to be important mechanisms causing neurological symptoms [5]. However, since there is poor correlation between ammonia and the severity of $\mathrm{HE}$, other factors could be implicated [6,7]. For instance, the cerebral accumulation of different substances besides ammonium, like amino acids (AA) [8], manganese [9], biliary salts, has been demonstrated in patients with cirrhosis or porto-systemic shunts. More recently, inflammation has been thought to contribute to the severity of HE both in animal models and also in humans $[6,10]$. Indeed, inflammation is clearly more marked in patients with ACLF and HE compared with those with acute decompensation but no ACLF with similar severities of $\mathrm{HE}$, suggesting that the underlying pathophysiological 
mechanisms may be different. Severe cerebral edema has been reported in some studies in cirrhotic patients with HE [11]. Indeed, death of about $5 \%$ ACLF patients is thought to be caused by severe cerebral edema [12]. Whether this is associated with blood-brain barriers dysfunction is not known.

Brain homeostasis is of major importance to carry out the complex physiological functions, especially synaptic transmission. Thus, exchanges between the blood and the brain are tightly regulated by tight junctions on two main cellular interfaces that restrict the permeability: the blood-brain barrier that is located on cerebral endothelial cells and the blood-cerebrospinal fluid barrier (BCSFB) located on epithelial cells of choroid plexuses (see $[13,23]$ for review). Even if tight junctions are expressed in the cerebral endothelial cells of the blood-brain barrier and on epithelial cells of choroid plexuses on the BCSFB, their molecular and cellular organization is very closely organized (see for review [13,14]). Dysfunction of these barriers have been recently highlighted in different neurological diseases and their dysfunction in cirrhotic patients, especially those with $\mathrm{HE}$, has been suggested to be pathophysiologically relevant $[11,14]$. Recent studies highlighted that the BCSFB constitute a large surface area for exchanges, greater than previously thought, and that this barrier intervene in availability of drugs. Thus, MRI studies have shown that in case of manganese accumulation for example, this metal first accumulates in CSF as a transfer across the choroid plexuses before spreading to periventricular regions where it enter into neural cells to reach the final destination via retrograde transport via axons [24]. Therefore, a more precise analysis of CSF in cirrhotic patients with or without ACLF would be of major interest but access to CSF is difficult since these patients often have low platelets that render lumbar puncture a high risk procedure. 
The measurement of the specific gravity (SG), which is defined as the weight of the volume of an object, is a validated and reliable method to characterize any solute, estimate the water content of CSF or of different brain regions $[15,16]$. CT scanning and post-processing analysis by a dedicated software, Brainview (Institut Nationale des Télécommunications $[15,17,18])$, has already been used to assess SG in patients for the evaluation of brain edema $[15,19,20]$. Thus, studying CSF-SG allows this question to be addressed. The normal CSF-SG is between 1.007 and 1.008 . Disease related variations of SG could be analyzed in patients that undergo a CT scan [20]. A decrease in CSF-SG suggests passage through the BCSFB of either a substances with a SG lower than that of the CSF or the passage of solute whereas an increase of CSF-SG suggests the passage through the BCSFB of a substances with a SG greater than that of the CSF. Interestingly, SG of the substances that has previously been shown as being present in the CSF of cirrhotic patients are well known and are as follows, ammonium has a SG of 0.9 , bile salts a SG between 1.010 and 1.040 and, immunoglobulins a SG of more than 1.060 .

Thus, the modification in the blood-CSF barrier permeability could represent a first step in the physiopathology of ACLF or HE, responsible for modification in the CSF composition and afterwards in the brain extracellular compartment.

Therefore, the aims of this study were to assess CSF-SG in acutely decompensated cirrhosis and to compare it in patients with and without ACLF and with and without HE. 


\section{PATIENTS AND METHODS}

\section{Patient's selection}

We retrospectively identified all the patients admitted for acute decompensation of cirrhosis in two Hepatology ICUs of two tertiary care centers (Royal Free Hospital, London, United-Kingdom and La Pitié-Salpêtrière Hospital, Paris, France) who underwent a brain CT-scan between January 2013 and April 2013. Both center admit about 250 patients every years for acute decompensation of cirrhosis and $50 \%$ of these patients present ACLF and 40\% present with HE. Those patients could present acute decompensation with or without ACLF [21] and with or without HE. Patients were included, provided they fulfilled the following criteria: (1) CT-scan with contiguous slices without interspaces; (2) no previous neurological history except HE; (3) no cerebral hemorrhage on CT; (4) no history of contrast medium injection within the last 2 weeks for analysis purpose. Patients were compared to a group of stable cirrhotic patients followed up in the same units who underwent brain CT-scan in their pre-transplant workup for hepatocellular carcinoma in order to rule out brain metastasis. Cirrhosis was diagnosed using a combination of clinical, biological, radiological, histological and anatomo-pathological features.

\section{Healthy controls}

Non-cirrhotic patients that underwent a CT-scan with contiguous slices without interspaces at emergency department for headaches or trauma and that were finally interpreted as normal were used as healthy controls.

\section{Data collection}

The following data were collected at admission: demographic data (age, gender), 
natural history of cirrhosis (etiology, presence of ascites, previous episodes of bleeding, previous episodes of $\mathrm{HE}$, hepatocellular carcinoma), cause of admission, physical examination, laboratory measurements (leukocyte and platelet count, prothrombin time (PT), international normalized ratio (INR), and levels of liver enzymes, bilirubin, creatinine, sodium, albumin and C-reactive protein (CRP)). The severity of cirrhosis was evaluated by the Child-Pugh score. The presence of HE and of ACLF was noted. The presence of HE was diagnosed as impairment in consciousness, or motor function in a patient with cirrhosis after exclusion of other causes of mental disturbance. The severity of HE was assessed by senior hepatologists using West-Haven score (WH). Overt HE was considered if WH was equal or higher than 2. To quantify organ dysfunction, the CLIF-OF score was used. This score derived from SOFA assess six organ systems (liver, kidneys, brain, coagulation, circulation, lungs) and takes in account the specificity of cirrhosis [21]. The presence or the absence and grade of ACLF was defined as previously described [22].

\section{CT-scan analysis}

CT-scans were acquired on a Philips scanner (Netherlands) in London and on a Siemens scanner (Germany) in Paris. Quantitative brain CT analysis was performed using the Brainview software (Institut National des Télécommunications) [17]. Briefly, this validated software $[17,18]$ is aimed to analyze DICOM images acquired from cerebral CT scans by performing automatical segmentation excluding extracranial compartments on each slices. Interactive slice-by-slice segmentation allows to delineate different anatomical regions. The software renders the weight, the volume 
and the specific gravity (SG) of the whole brain and of each individual region (right and left hemispheres, brainstem, cerebellum, and intraventricular CSF).

\section{Statistical analysis}

Continuous variables are expressed as median and interquartile range (IQR). Categorical variables are expressed as frequencies and percentages. Chi-square or Fisher exact test was used to compare categorical variables and ANOVA for continuous variables, with Tukey range test for correction. Univariate analysis was used to compare the variables between the different groups. In order to take in account the acquisition in two centers, and the possible differences in CT calibration, stratification according to the center was performed.

All statistical tests were two-tailed. $P$ values that were less than 0.05 were considered to indicate statistical significance. Analysis involved use of JMP v9.0 (SAS Inst., Cary, NC).

According to the French law, the approval of the local ethics committee was not necessary since the CTs were included in the clinical management of the patients and the study was retrospective. The database in which the patients were identified had been submitted to and approved by the French national commission for computerized files and liberty (CNIL, Commission Nationale de l'Informatique et des Liberte's). Royal Free Hospital research ethics committee gave its approval for the study. 


\section{RESULTS}

\section{Subjects}

From January 2013 to April 2013, 72 cirrhotic patients underwent a cerebral CT scan.

Five CTs were not analyzable, thus 67 patients were finally retained for analysis. Overall, 36 cirrhotic patients were admitted for acute decompensation ( 25 presented with ACLF and 11 with acute decompensation without ACLF) and 31 for stable cirrhosis. Results were compared to that of 61 healthy controls. Baseline characteristics of the patients and the controls are displayed in Table 1. In patients with acute decompensation, 20 patients $(56 \%)$ displayed overt HE (WH grade 2: $\mathrm{n}=12$, grade $3, \mathrm{n}=7$, grade $4, \mathrm{n}=1)$ and $25(69 \%)$ displayed ACLF. 15 patients $(42 \%)$ presented with both ACLF and HE. No patients had HE or ACLF in the stable cirrhotic patients. Indication for brain CT-scan differed significantly between patients with and without overt HE. Brain CT-scans in healthy controls were performed mainly because of headache.

\section{Quantitative CT analysis}

SG values did not differ between the two centers. Thus, data were analyzed together.

Cirrhotic patients whether they were or not decompensated display increased CSF$S G$ as compared to healthy controls

Compared to healthy controls, cirrhotic patients had increased CSF-SG (1.01178 [1.01014-1.01375] vs 1.00855 [1.00688-1.01045], $+0.3 \%, p<0.0001$, respectively) suggesting the passage of substances with a SG greater than that of the CSF through the BCSFB in cirrhotic patients. 
Acutely decompensated cirrhotic patients display a mild decrease in CSF-SG as compared to patients with stable cirrhosis

Acutely decompensated cirrhotic patients compared to stable cirrhotic patients had a decreased CSF-SG (1.01088 [1.00922-1.01224] vs 1.01256 [1.01156-1.01458] respectively, $-0.2 \%, p=0.0002$ ) suggesting increased permeability of the BCSFB to either solutes or substances with a lower SG in this setting (Table 2 and Figure $1 \mathrm{~A}$ ). CSF-SG in acutely decompensated cirrhotic patients remained however higher than in healthy controls $(1.01088$ [1.00922-1.01224] vs 1.00855 [1.00688-1.01045], respectively, $+0.2 \%, p<0.0001)$.

Cirrhotic patients with ACLF have decreased CSF-SG as compared to cirrhotic patients without $A C L F$

Cirrhotic patients with ACLF displayed decreased CSF-SG as compared to patients without ACLF (1.01036 [1.00908-1.01207] vs 1.01218 [1.01103-1.01412] respectively, $-0.2 \%, p=0.0030$,), suggesting increased permeability of the BCSFB to either solutes or substances with a lower SG in this setting (Table 3 and Figure 1B).

The presence of HE does not modify the CSF-SG in cirrhotic patients.

In cirrhotic patients, patients with HE displayed a CSF-SG of 1.01122 [1.009861.01224] compared to 1.01209 [1.01024-1.01408], $-0.09 \%$, in patients without HE $(p=0.1757)$ (Table 3 and Figure 1C). 


\section{DISCUSSION}

By using CT scanning analysis of the SG of the CSF, we found arguments in favor of increased permeability of the BCSFB in cirrhotic patients. Indeed, results of the present pilot study suggest that cirrhotic patients display alterations of the BCSFB, that are dependent on the severity of liver disease. Importantly, these alterations are influenced by the presence of ACLF, but occur even in the absence of HE. These results suggest that, in cirrhotic patients, BCSFB permeability to substances and solutes are altered.

It seems that, early in cirrhosis, even in those without acute decompensation, some substances with a SG higher than that of the CSF, i.e. higher than 1.008 , cross the BCSFB. Elevated levels of glutamine, glutamate, alpha ketoglutarate, bile salts and increased concentrations of serotonin, dopamine and AA have been reported in the CSF of cirrhotic patients [26-28]. According to our results in stable cirrhotic patients compared to healthy controls, the passage of bile salts, the SG of which ranges from 1.010 to 1.040 , into the CSF could explain the increase in CSF-SG. Indeed, bile salts have been shown to accumulate in these conditions [29]. Furthermore, bile salts have a synergistic effect with endotoxin and ammonia on the formation of brain edema by their action on Na-K-ATPase [30]. Several data also reported an increased level in both brain and CSF of tryptophan and its metabolites 5-HIAA, in patients with porto-systemic shunts and cirrhosis [31]. Hence, in cirrhotic patients the level of tryptophan in the CSF was 4 -fold higher than in controls. Several AA were found to be elevated in the CSF in the setting of ALF [32], and animal models of cirrhosis displayed an increased CSF level of tryptophan [31]. In the CSF of cirrhotic patients without $\mathrm{HE}$, a significant increase was found in nearly all amino acids, including those 
known not to cross the blood-brain barriers readily, suggesting the presence of a nonspecific modification of the BCSFB permeability [28]. This could also explain the increase of CSF-SG in the cirrhotic patients. The passage of proteins such as IgG seems less probable since no major brain edema was present. The possible passage of some yet unknown substance or medication cannot be ruled out. Indeed, increase of CSF-SG is a classical finding in the administration of local anesthesics for spinal anaesthesia. In some pathological conditions such as seizures, increased blood osmolarity can induce dehydration of the brain and thus an increase its SG [33]. We recently showed using metabolomics that the CSF of cirrhotic patients displaying neurological symptoms contained the main above mentioned substances and several xenobiotics, i.e. drugs, that all could contribute to the modification in CSF-SG $[34,35]$.

In cirrhotic patients with HE, CSF-SG was not statistically different compared to cirrhotic patients without HE. This observation is somewhat surprising given that ammonia (SG: 0.9) has been shown to be increased in the CSF in patients with HE [36]. The data support the hypothesis that alterations of cerebral homeostasis occur even in the absence of $\mathrm{HE}$, evidenced by studies showing alterations in myoinositol and glutamine in the brain of patients with cirrhosis with and without $H E[37,38]$. The manifestation of $\mathrm{HE}$ is therefore likely to be due not only to alterations of blood-brain barriers, but also to other interacting factors.

In cirrhotic patients with ACLF, CSF-SG was decreased compared to acute decompensation without ACLF patients. This finding suggests again an alteration in BCSFB. Inflammation is the main physiopathological hallmark of ACLF. Hence, one hypothesis is an increased passage of solutes since BCSFB permeability to solutes, 
is particularly influenced by inflammatory mediators such as TNF-alpha or IFNgamma. Our results suggest that ACLF patients present altered BCSFB even in the absence of HE. The significance of this observation is not clear. It is tempting to hypothesize that these abnormalities may make the patient susceptible to the development of HE but this hypothesis will have to be confirmed in future studies.

Our study has several limitations. First, it was retrospective and the diagnosis of ACLF and HE was retrieved in medical records. Bias could be introduced in our study by the fact that we considered only overt HE and not minimal HE. Since this retrospective design, case-mix cannot be excluded. For example, patients who underwent a CT-scan for another reason than HE were patients hospitalized for the workup of hepatocellular carcinoma, which were displayed far more severe liver disease than usual patients with hepatocellular carcinoma. Furthermore, we could include only patients that underwent CTs without interslice spaces. Last, the composition of substances that cross the BCSFB need further analysis of the CSF but lumbar puncture is rarely indicated in cirrhotic patients, and this type of study is probably not ethically possible. This study provides the novel data describing changes in CSF content in cirrhotic patients.

In conclusion, our study describes for the first time that CSF-SG is modified in cirrhotic patients, both with and without HE suggesting altered BCSFB permeability to substances and solutes. Patients with ACLF display further alterations of BCSFB, which may indicate their susceptibility to HE. The observation that abnormalities in the BCSFB are manifest even in the absence of overt HE may have important clinical and pathophysiological implications. 
Acknowledgement: The authors gratefully acknowledge the help of Dr Dominic Yu, Consultant Radiologist at Royal Free Hospital.

Financial Support: none

\section{List of abbreviations}

\begin{tabular}{|c|l|}
\hline ACLF & Acute-on chronic liver failure \\
\hline AA & Amino-acids \\
\hline ALF & Acute liver failure \\
\hline BCSFB & Blood-cerebrospinal fluid barrier \\
\hline CSF & Cerebrospinal fluid \\
\hline CRP & C-reactive protein \\
\hline HE & Hepatic encephalopathy \\
\hline INR & International normalized ratio \\
\hline MRI & Magnetic resonance imaging \\
\hline MELD & Model for End-Stage Liver Disease \\
\hline PT & Prothrombin time \\
\hline SG & Specific gravity \\
\hline WH & West-Haven score \\
\hline
\end{tabular}

\section{Conflict of interest}

Nicolas Weiss declares the following potential conflicts of interest.

Research grant: Eumedica, Gilead

Speaker fees: Alpha-Wasserman, Norgine, Gore

Dominique Thabut declares the following potential conflicts of interest. Research grant: Eumedica, Gilead. Speaker fees: Alpha-Wasserman, Norgine, Gore, Gilead, BMS, Janssen 
Rajiv Jalan declares the following potential conflicts of interest. Inventor: Ornithinephenylacetate, a treatment for hepatic encephalopathy; UCL Liver dialysis device. Research grant: Ocera therapeutics, Gambro, Sequana and Grifols. Consultant: Ocera therapeutics, Conatus. Founder: Yaqrit Itd. (UCL spin out). Speaker fees: Grifols, Norgine.

The other authors have nothing to declare. 


\section{REFERENCES}

1. Stepanova M, Mishra A, Venkatesan C, Younossi ZM. In-hospital mortality and economic burden associated with hepatic encephalopathy in the United States from 2005 to 2009. Clin Gastroenterol Hepatol Off Clin Pract J Am Gastroenterol Assoc. 2012;10:1034-1041.e1.

2. Orr JG, Homer T, Ternent L, et al. Health related quality of life in people with advanced chronic liver disease. J Hepatol. 2014;61:1158-1165.

3. Cordoba J, Ventura-Cots M, Simón-Talero M, et al. Characteristics, risk factors, and mortality of cirrhotic patients hospitalized for hepatic encephalopathy with and without acute-on-chronic liver failure (ACLF). J Hepatol. 2014;60:275-281.

4. Bajaj JS, Schubert CM, Heuman DM, et al. Persistence of cognitive impairment after resolution of overt hepatic encephalopathy. Gastroenterology. 2010;138:2332-2340.

5. Romero-Gómez M. Role of phosphate-activated glutaminase in the pathogenesis of hepatic encephalopathy. Metab Brain Dis. 2005;20:319-325.

6. Shawcross DL, Davies NA, Williams R, Jalan R. Systemic inflammatory response exacerbates the neuropsychological effects of induced hyperammonemia in cirrhosis. J Hepatol. 2004;40:247-254.

7. Ong JP, Aggarwal A, Krieger D, et al. Correlation between ammonia levels and the severity of hepatic encephalopathy. Am J Med. 2003;114:188-193.

8. James JH, Escourrou J, Fischer JE. Blood-brain neutral amino acid transport activity is increased after portacaval anastomosis. Science. 1978;200:1395-1397.

9. Rose C, Butterworth RF, Zayed J, et al. Manganese deposition in basal ganglia structures results from both portal-systemic shunting and liver dysfunction. Gastroenterology. 1999;117:640-644.

10. Wright G, Davies NA, Shawcross DL, et al. Endotoxemia produces coma and brain swelling in bile duct ligated rats. Hepatol Baltim Md. 2007;45:1517-1526.

11. Jalan R, Bernuau J. Induction of cerebral hyperemia by ammonia plus endotoxin: does hyperammonemia unlock the blood-brain barrier? J Hepatol. 2007;47:168-171.

12. Joshi D, O'Grady J, Patel A, et al. Cerebral oedema is rare in acute-on-chronic liver failure patients presenting with high-grade hepatic encephalopathy. Liver Int Off J Int Assoc Study Liver. Epub 2013 Jun 20.

13. Ghersi-Egea JF, Leininger-Muller B, Cecchelli R, Fenstermacher JD. Bloodbrain interfaces: relevance to cerebral drug metabolism. Toxicol Lett. 1995;8283:645-653.

14. Weiss N, Miller F, Cazaubon S, Couraud P-O. The blood-brain barrier in brain homeostasis and neurological diseases. Biochim Biophys Acta. 2009;1788:842-857.

15. Lescot T, Bonnet M-P, Zouaoui A, et al. A quantitative computed tomography assessment of brain weight, volume, and specific gravity in severe head trauma. Intensive Care Med. 2005;31:1042-1050.

16. Lescot T, Degos V, Puybasset L. Does the brain become heavier or lighter after trauma? Eur J Anaesthesiol Suppl. 2008;42:110-114.

17. Puybasset L, Cluzel P, Chao N, Slutsky AS, Coriat P, Rouby JJ. A computed tomography scan assessment of regional lung volume in acute lung injury. The CT Scan ARDS Study Group. Am J Respir Crit Care Med. 1998;158:1644-1655.

18. Vieira SR, Puybasset L, Richecoeur J, et al. A lung computed tomographic assessment of positive end-expiratory pressure-induced lung overdistension. Am J Respir Crit Care Med. 1998;158:1571-1577. 
19. Degos V, Lescot $T$, Icke $C$, et al. Computed tomography-estimated specific gravity at hospital admission predicts 6 -month outcome in mild-to-moderate traumatic brain injury patients admitted to the intensive care unit. Anesth Analg.

2012;114:1026-1033.

20. Lescot T, Degos V, Zouaoui A, Préteux F, Coriat P, Puybasset L. Opposed effects of hypertonic saline on contusions and noncontused brain tissue in patients with severe traumatic brain injury. Crit Care Med. 2006;34:3029-3033.

21. Jalan R, Saliba F, Pavesi M, et al. Development and validation of a prognostic score to predict mortality in patients with acute-on-chronic liver failure. J Hepatol. 2014;61:1038-1047.

22. Moreau R, Jalan R, Gines $P$, et al. Acute-on-chronic liver failure is a distinct syndrome that develops in patients with acute decompensation of cirrhosis.

Gastroenterology. 2013;144:1426-1437, 1437.e1-e9.

23. Ghersi-Egea J-F, Strazielle N. Choroid plexus transporters for drugs and other xenobiotics. J Drug Target. 2002;10:353-357.

24. Aoki I, Naruse S, Tanaka C. Manganese-enhanced magnetic resonance imaging (MEMRI) of brain activity and applications to early detection of brain ischemia. NMR Biomed. 2004;17:569-580.

25. Degos V, Lescot T, Zouaoui A, et al. Computed tomography-estimated specific gravity of noncontused brain areas as a marker of severity in human traumatic brain injury. Anesth Analg. 2006;103:1229-1236.

26. Jellinger K, Riederer P, Rausch WD, Kothbauer P. Brain monoamines in hepatic encephalopathy and other types of metabolic coma. J Neural Transm Suppl. Epub 1978.:103-120.

27. Ono J, Hutson DG, Dombro RS, Levi JU, Livingstone A, Zeppa R. Tryptophan and hepatic coma. Gastroenterology. 1978;74:196-200.

28. Cascino A, Cangiano C, Fiaccadori F, et al. Plasma and cerebrospinal fluid amino acid patterns in hepatic encephalopathy. Dig Dis Sci. 1982;27:828-832.

29. Gove CD, Hughes RD, Ede RJ, Williams R. Regional cerebral edema and chloride space in galactosamine-induced liver failure in rats. Hepatol Baltim Md. 1997;25:295-301.

30. Tominaga S, Watanabe A, Tsuji T. Synergistic effect of bile acid, endotoxin, and ammonia on brain edema. Metab Brain Dis. 1991;6:93-105.

31. Young SN, Lal S, Sourkes TL, Feldmuller F, Aronoff A, Martin JB.

Relationships between tryptophan in serum and CSF, and 5-hydroxyindoleacetic acid in CSF of man: effect of cirrhosis of liver and probenecid administration. J Neurol Neurosurg Psychiatry. 1975;38:322-330.

32. Mattson LR, Lindor NM, Goldman DH, Goodwin JT, Groover RV, Vockley J. Central pontine myelinolysis as a complication of partial ornithine carbamoyl transferase deficiency. Am J Med Genet. 1995;60:210-213.

33. Nitsch C, Fujiwara K, Kuroiwa T, Klatzo I. Specific gravity increases and brain water content decreases during short epileptiform seizures in discrete rabbit brain areas. J Neurol Sci. 1984;64:119-129.

34. Weiss N, Junot C, Rudler M, Thabut D. Hepatic versus drug-induced encephalopathy in cirrhotic patients? Liver Int Off J Int Assoc Study Liver. Epub 2016 Jan 20.

35. Weiss N, Barbier de Saint Hilaire P, Colsch B, et al. Cerebrospinal fluid metabolomics highlights dysregulation of energy metabolism in overt hepatic encephalopathy. J Hepatol. in press.

36. Ropper A, Samuels M, Klein J. Adams and Victor's Principles of Neurology 
10th Edition. 10 edition. New York: McGraw-Hill Professional; 2014.

37. Chavarria L, Alonso J, García-Martínez R, et al. Brain magnetic resonance spectroscopy in episodic hepatic encephalopathy. J Cereb Blood Flow Metab Off J Int Soc Cereb Blood Flow Metab. 2013;33:272-277.

38. Rovira A, Grivé E, Pedraza S, Rovira A, Alonso J. Magnetization transfer ratio values and proton MR spectroscopy of normal-appearing cerebral white matter in patients with liver cirrhosis. AJNR Am J Neuroradiol. 2001;22:1137-1142. 
Table 1: Baseline characteristics of the patients and the controls.

\begin{tabular}{|c|c|c|c|c|}
\hline & $\begin{array}{l}\text { Acute decompensation } \\
\text { of cirrhosis }\end{array}$ & Stable cirrhotic patients & Healthy controls & $p$ value \\
\hline & $n=36$ & $\mathrm{n}=31$ & $\mathrm{n}=61$ & \\
\hline Age, years & 55 [47-65] & 59 [48-63] & 64 [46-78] & 0.3347 \\
\hline Male gender (\%) & $23(64 \%)$ & $26(84 \%)$ & $39(64 \%)$ & 0.1134 \\
\hline \multicolumn{5}{|l|}{ Etiology of cirrhosis } \\
\hline Viral & $4(11 \%)$ & $0(0 \%)$ & - & \\
\hline Alcohol consumption & $22(61 \%)$ & $22(72 \%)$ & - & \\
\hline Other & $10(28 \%)$ & $8(25 \%)$ & - & \\
\hline Ascites & $9(56 \%)$ & $0(0 \%)$ & - & $<0.0001$ \\
\hline ACLF & $25(69 \%)$ & $0(0 \%)$ & - & $<0.0001$ \\
\hline Hepatocelullar & $3(8 \%)$ & $31(100 \%)$ & - & $<0.0001$ \\
\hline \multicolumn{5}{|l|}{ carcinoma } \\
\hline \multicolumn{5}{|l|}{ hepatitis } \\
\hline TIPS & $7(44 \%)$ & - & - & \\
\hline Asterixis & $11(69 \%)$ & $0(0 \%)$ & - & $<0.0001$ \\
\hline PTT (\%) & $32[31-44]$ & 64 [46-76] & NA & 0.0001 \\
\hline INR & $2.0[1.6-2.5]$ & $1.4[1.2-2.0]$ & NA & 0.0081 \\
\hline $\begin{array}{l}\text { Leukocyte count } \\
\text { (/mm3) }\end{array}$ & 9625 [6 683-16 848] & 4900 [3 450-7 800] & 10030 [7140-12 920] & 0.0055 \\
\hline Platelet count (/mm3) & 81500 [50 500-125 750] & 67000 [47 500-88 500] & NA & 0.3442 \\
\hline Creatinine level (mg/dL) & 0.94 [0.63-1.35] & 0.74 [0.67-0.89] & NA & 0.1309 \\
\hline Albumin level ( $\mathrm{g} / \mathrm{L})$ & 25 [22-31] & $34[31-40]$ & NA & 0.0014 \\
\hline Bilirubin level (mg/dL) & $5.1[1.7-8.9]$ & $1.1[0.8-1.9]$ & NA & 0.0013 \\
\hline AST level (U/L) & $82[60-177]$ & $67[46-82]$ & NA & 0.0931 \\
\hline ALT level (U/L) & $48[25-80]$ & $45[30-55]$ & NA & 0.3700 \\
\hline Sodium level (mmol/L) & 139 [133-145] & 139 [134-142] & NA & 0.3339 \\
\hline CRP level (mg/l) & $20.5[6.3-48.3]$ & $7.5[4.0-14.0]$ & NA & 0.0203 \\
\hline \multicolumn{5}{|l|}{ Child-Pugh score } \\
\hline$A$ & $2(6 \%)$ & $26(83 \%)$ & - & \\
\hline B & $2(6 \%)$ & $5(17 \%)$ & - & \\
\hline $\mathrm{C}$ & 32 (88\%) & 0 & - & \\
\hline CLIF-SOFA score & $11[8-12]$ & NA & - & \\
\hline Hepatic & $20(56 \%)$ & $0(0 \%)$ & & $<0.0001$ \\
\hline $\begin{array}{l}\text { encephalopathy } \\
\text { West-Haven score }\end{array}$ & & & & $<0.0001$ \\
\hline - & $4(11 \%)$ & $31(100 \%)$ & - & \\
\hline 1 & $12(33 \%)$ & - & - & \\
\hline 2 & 12 (33\%) & - & - & \\
\hline 3 & $7(19 \%)$ & - & - & \\
\hline 4 & $1(3 \%)$ & - & - & \\
\hline
\end{tabular}

Data are mean \pm SD or number (\%).

Abbreviations: $A L T$, alanine aminotransferase; $A S T$, aspartate aminotransferase; $C R P, C$-reactive protein; HE, hepatic encephalopathy; INR, international standardized ratio; MELD, Model for EndStage Liver Disease; PTT, prothrombin time 
Table 2: Specific gravity of different brain regions according to the presence or not of cirrhosis and the presence or not of acute decompensation of cirrhosis.

\begin{tabular}{|c|c|c|c|c|}
\hline & $\begin{array}{c}\text { Healthy controls } \\
\mathrm{n}=61\end{array}$ & $\begin{array}{c}\text { Stable cirrhotic patients } \\
\mathrm{n}=31\end{array}$ & $\begin{array}{c}\text { Acute decompensation of } \\
\text { cirrhosis } \\
\mathrm{n}=36\end{array}$ & $\mathbf{p}$-value \\
\hline CSF & $1.00855[1.00688-1.01045]$ & $1.01256[1.01156-1.01450]$ & $1.01088[1.00922-1.01224]$ & $<\mathbf{0 . 0 0 0 1}$ \\
\hline $\begin{array}{c}\text { Relative } \\
\text { difference }(\%)\end{array}$ & $\mathrm{na}$ & $+0.4 \%$ & $+0.2 \%$ & \\
\hline Whole brain & $1.02934[1.02812-1.03108]$ & $1.02920[1.02792-1.03023]$ & $1.02844[1.02708-1.02967]$ & $\mathbf{0 . 0 3 2 9}$ \\
\hline $\begin{array}{c}\text { Relative } \\
\text { difference }(\%)\end{array}$ & $\mathrm{na}$ & $-0.01 \%$ & $-0.08 \%$ & \\
\hline Hemispheres & $1.02903[1.02753-1.03058]$ & $1.02885[1.02766-1.02965]$ & $1.02793[1.02657-1.02940]$ & $\mathbf{0 . 0 4 6 2}$ \\
\hline $\begin{array}{c}\text { Relative } \\
\text { difference }(\%)\end{array}$ & $\mathrm{na}$ & $-0.01 \%$ & $-0.1 \%$ & \\
\hline Brainstem & $1.02138[1.02080-1.02231]$ & $1.02534[1.02373-1.02593]$ & $1.02448[1.02290-1.02580]$ & $<\mathbf{0 . 0 0 0 1}$ \\
\hline $\begin{array}{c}\text { Relative } \\
\text { difference }(\%)\end{array}$ & $\mathrm{na}$ & $+0.4 \%$ & $+0.3 \%$ & \\
\hline Cerebellum & $1.03502[1.03310-1.03619]$ & $1.03297[1.03081-1.03360]$ & $1.03201[1.03114-1.03430]$ & $<\mathbf{0 . 0 0 0 1}$ \\
\hline $\begin{array}{c}\text { Relative } \\
\text { difference }(\%)\end{array}$ & $\mathrm{na}$ & $-0.3 \%$ & $-0.3 \%$ & \\
\hline
\end{tabular}

Relative differences are given compared to controls.

Abbreviation: CSF, cerebrospinal fluid; na: not applicabla.

Table 3: Specific gravity of different brain regions according to the presence or absence of ACLF and HE.

\begin{tabular}{|c|c|c|c|c|c|c|}
\hline & \multicolumn{2}{|c|}{ Hepatic encephalopathy } & \multirow[b]{2}{*}{ p-value } & \multicolumn{2}{|c|}{ ACLF } & \multirow[b]{2}{*}{ p-value } \\
\hline & $\begin{array}{c}\text { No } \\
n=47\end{array}$ & $\begin{array}{c}\text { Yes } \\
n=20\end{array}$ & & $\begin{array}{c}\text { No } \\
n=42\end{array}$ & $\begin{array}{c}\text { Yes } \\
n=25\end{array}$ & \\
\hline CSF & $\begin{array}{c}1.01209[1.01024- \\
1.01408]\end{array}$ & $\begin{array}{c}1.01122[1.00986- \\
1.01224]\end{array}$ & 0.1757 & $\begin{array}{c}1.01218[1.01103- \\
1.01412]\end{array}$ & $\begin{array}{c}1.01036[1.00908- \\
1.01207]\end{array}$ & 0.0030 \\
\hline $\begin{array}{r}\text { Relative } \\
\text { difference (\%) }\end{array}$ & $\mathrm{Na}$ & $-0.09 \%$ & & na & $-0.2 \%$ & \\
\hline Whole brain & $\begin{array}{c}1.02940[1.02782- \\
1.03023]\end{array}$ & $\begin{array}{c}1.02854[1.02718- \\
1.02966]\end{array}$ & 0.4353 & $\begin{array}{c}1.02912[1.02781- \\
1.03014]\end{array}$ & $\begin{array}{c}1.02866[1.02669- \\
1.02975]\end{array}$ & 0.1450 \\
\hline $\begin{array}{r}\text { Relative } \\
\text { difference (\%) }\end{array}$ & na & $-0.08 \%$ & & na & $-0.05 \%$ & \\
\hline Hemispheres & $\begin{array}{c}1.02871[1.02737- \\
1.02968]\end{array}$ & $\begin{array}{c}1.02812[1.02663- \\
1.02942]\end{array}$ & 0.3946 & $\begin{array}{c}1.02873[1.02747- \\
1.02965]\end{array}$ & $\begin{array}{c}1.02838[1.02613- \\
1.02960]\end{array}$ & 0.1255 \\
\hline $\begin{array}{r}\text { Relative } \\
\text { difference (\%) }\end{array}$ & na & $-0.06 \%$ & & na & $-0.03 \%$ & \\
\hline Brainstem & $\begin{array}{c}1.02494[1.02342- \\
1.02571]\end{array}$ & $\begin{array}{c}1.02487[1.02305- \\
1.02630]\end{array}$ & 0.7815 & $\begin{array}{c}1.02515[1.02344- \\
1.02572]\end{array}$ & $\begin{array}{c}1.02479[1.02292- \\
1.02618]\end{array}$ & 0.6563 \\
\hline $\begin{array}{r}\text { Relative } \\
\text { difference (\%) }\end{array}$ & na & $-0.01 \%$ & & na & $-0.04 \%$ & \\
\hline Cerebellum & $\begin{array}{c}1.03266[1.03083- \\
1.03360]\end{array}$ & $\begin{array}{c}1.03256[1.03130- \\
1.03455]\end{array}$ & 0.3952 & $\begin{array}{c}1.03268[1.03079- \\
1.03359]\end{array}$ & $\begin{array}{c}1.03226[1.03122- \\
1.03443]\end{array}$ & 0.6648 \\
\hline $\begin{array}{r}\text { Relative } \\
\text { difference (\%) }\end{array}$ & na & $-0.01 \%$ & & na & $-0.04 \%$ & \\
\hline
\end{tabular}

Relative differences are given compared to controls.

Abbreviation: ACLF, acute on chronic liver failure ; CSF, cerebrospinal fluid. 


\section{FIGURES LEGENDS}

Figure 1: CSF specific gravity according to the presence or not of cirrhosis, the presence or not of acute decompensation of cirrhosis, of ACLF and HE.

A, CSF SG according to the presence or not of cirrhosis and the presence or not of acute decompensation of cirrhosis; B, CSF SG according to the presence or not of ACLF; C, CSF SG according to the presence or not of HE.

Supplemental figure: Specific gravity of different brain regions according to the presence or not of cirrhosis and the presence or not of acute decompensation of cirrhosis. 
A

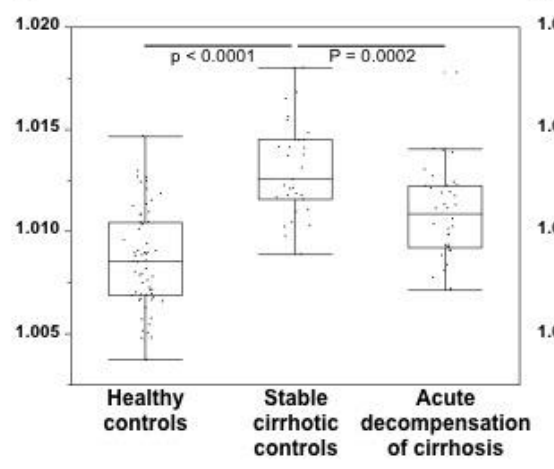

B

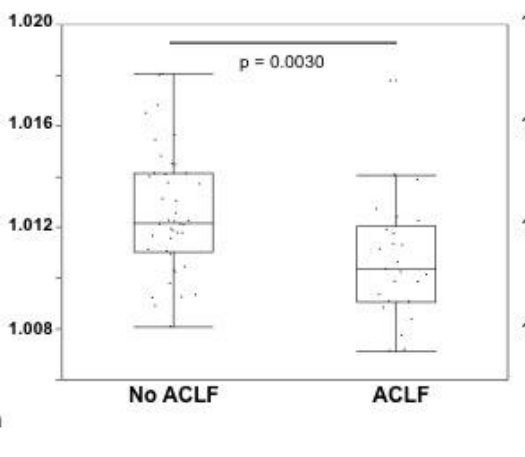

C

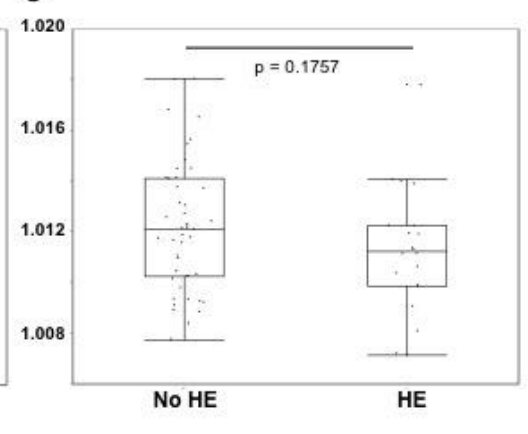

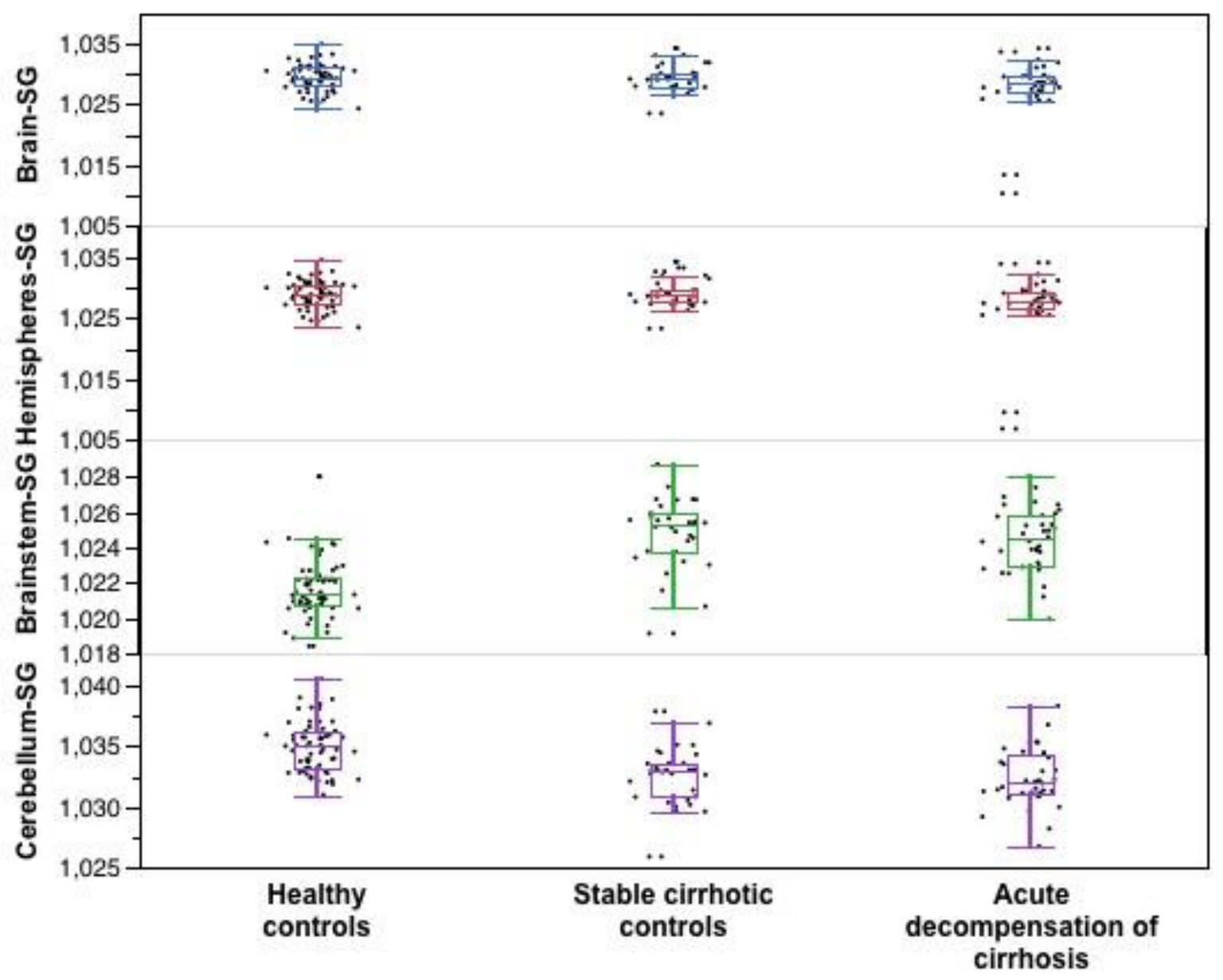

